\title{
Pericardial biopsy and fenestration
}

\author{
R. INDERBITZI, M. FURRER AND F. LeUPI
}

Department of Thoracic and Cardiovascular Surgery, University of Berne. Switzerland

KEY WORDS: Pericardial effusion, thoracoscopic fenestration, diagnostic, treatment, minimally invasive surgery.

Employing a video thoracoscopic pericardial fenestration constitutes a promising technique for the investigation and treatment of chronic pericardial effusions. It combines the benefit of low invasiveness with the advantages of open biopsy. The procedure simultaneously allow's both an accurate diagnosis under visual control (inspection, aspiration, welltargeted biopsy of pathological processes) and the performance of effective therapeutic intervention. Without imposing unacceptable stress, it also facilitates rapid symptom relief in patients with advanced malignant disease whose general condition is severely impaired.

\section{Introduction}

The treatment of persistent or recurrent pericardial effusion depends on the underlying disease and on clinical symptoms. When there is no response to initial antiinflammatory treatment with non-steroidal drugs given under strict clinical control, the next diagnostic step is aspiration under echocardiographic control followed, if the results are negative or unclear, by sub-xiphosternal open biopsy. A large range of treatments can be employed, extending from conservative concepts via pericardiocentesis ${ }^{[1]}$, thoracoscopic pericardial fenestration ${ }^{[2]}$ and open sub-xiphosternal pericardiotomy ${ }^{[3]}$, to radical pericardectomy.

This paper deals with thoracoscopic fenestration assisted by video-endoscopy. Owing to the transmission of visual information from the video camera to a monitor, this method combines the advantage of minimal invasiveness with the possibilities of surgical techniques.

\section{Case histories}

CASE I

A 45-year-old woman was referred for diagnostic and therapeutic pericardial fenestration, because of a persistent pericardial effusion and pericardial thickening associated with cardiomegaly of uncertain origin known since 1980. This procedure was performed on 29 August 1990 under endotracheal anaesthesia using the doublelumen technique. Three hundred millilitres of effusion fluid (cytologically and bacteriologically negative) were withdrawn and a pericardial window was made measuring $4 \times 4 \mathrm{~cm}$ (histology: chronic connective tissue proliferation). The postoperative course was uneventful. The drain could be withdrawn after $24 \mathrm{~h}$ and postoperative hospitalization lasted 3 days. Eleven months later, the patient was symptom-free without medication and follow-up echocardiography showed normal cardiac performance.

Submitted for publication on 3 January 1992, and in revised form 10 July 1992

Correspondence - R. Inderbitzi, MD. Dept. of Thoracic and Cardiovascular Surgery, Lniversity of Berne, 3010 Berne, Switzerland.
CASE 2

A 64-year-old man underwent an upper lobe resection of the left lung in 1984 after discovery of an adenocarcinoma. On 9 April 1991, a pericardial effusion had to be evacuated as an emergency due to acute pericardial tamponade. Cytology of the effusion fluid revealed adenocarcinoma cells consistent with the histology of the lung carcinoma removed in 1984 . Recurrence of pericardial effusion required thoracoscopic pericardial fenestration. The pre-operative computer tomogram demonstrated thickening of the bronchial walls in the left lower lobe, of the pericardium and of the left pleura. Due to the presence of pleural scarring, palliative pericardial fenestration was performed through the right pleural cavity under general anaesthesia using the double-lumen tube. Intra-operatively, $500 \mathrm{ml}$ of a blood-tinged effusion fluid was again withdrawn from the pericardial cavity. Through a T-shaped incision, a triangular-shaped window measuring $5 \times 4 \times 4 \mathrm{~cm}$ was created by removal of a corresponding pericardial flap. Postoperative drainage continued for $24 \mathrm{~h}$, and the patient was discharged after 4 days. Two months later the patient was free of cardiac symptoms and shows no signs of cardiomegaly.

CASE 3

A 65-year-old patient with recurrent myopericarditis showed signs compatible with pericardial effusion at echocardiography. To confirm the diagnosis, a left-sided thoracoscopic pericardial biopsy was planned under endotracheal anaesthesia using the double-lumen technique. Endoscopic exploration demonstrated that pericardial movements were synchronous with the heart action. The thickened pericardium could not be grasped with the endoscopy forceps. Open transthoracic perimyocardial biopsy was then performed showing complete obliteration of the pericardical space in the area visualised by endoscopy. The histological preparation revealed the diagnosis of active, non-specific, fibrinous, constrictive pericarditis. One month later, peri-epicardectomy had to be carried out in order to release the severly constricted right ventricle. 


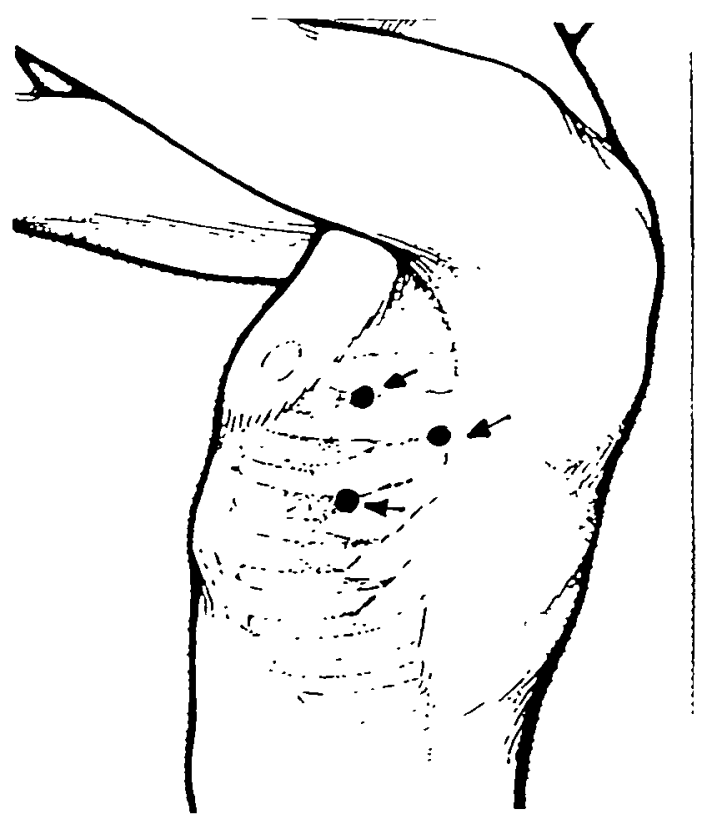

Figure 1 Portal placements.

\section{Operative technique}

The operation is performed with the patient in the lateral position under general anaesthesia using a doublelumen endobronchial tube. Three incisions are made in the axillary triangle formed by the axilla, the lower border of the major pectoralis muscle and the anterior border of the latissimus dorsi muscle, in the third, fourth and fifth intercostal spaces, with a distance of at least $6 \mathrm{~cm}$ between each portal (Fig. 1). A trocar is placed into the first skin incision and then the straight telescope connected to the video camera is inserted. The endoscopic procedure is transmitted to a TV monitor. The other two trocars are then inserted with the aid of endoscopic viewing. After induction of the pneumothorax, routine diagnostic inspection of the whole thoracic cavity is carried out. Any pathological finding should be submitted to biopsy. The external surface of the pericardium is inspected and the phrenic nerve identified. Fenestration is planned anteriorly or posteriorly to the nerve, depending on its anatomical course. The pericardium is carefully grasped and elevated using atraumatic forceps. After incision of the pericardial sac using scissors or a scalpel with a rounded tip, the effusion fluid is carefully evacuated and sent for bacteriological, cytological and chemical analysis. Suction of the epicardium should be avoided to prevent serious disturbances of cardiac rhythm. Incidental supraventricular extrasystoles due to surgical manipulation can, however, hardly be averted. Following opening of the pericardial cavity, a flap of the desired size is prepared (Fig. 2). The fenestration terminates after a meticulous check for haemostasis. Through the created window, a considerable section of the pericardial cavity can be carefully sounded and explored for adhesions, using an angled, blunt probe. At the end of the procedure a (24-gauge Charriere) chest tube is inserted through the

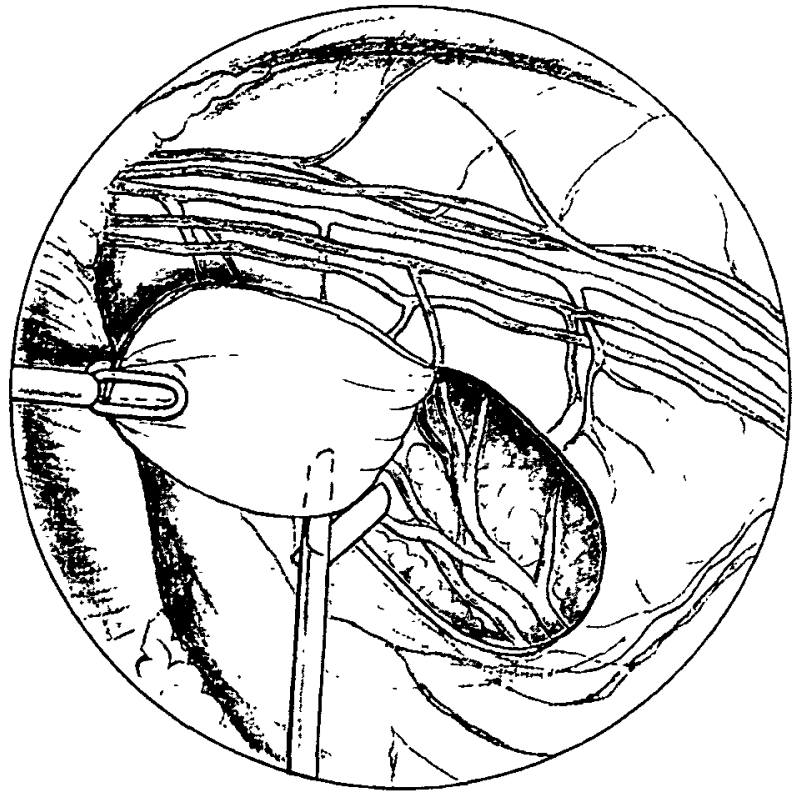

Figure 2 Endoscopic view. A pericardial flap is created using forceps and scissors. Note the diaphragm (on the left), the mediastinum (on the top) and the pericardium with the phrenic nerve.

most distal incision of the thoracic wall and positioned under visual control with the tip ventro-basally in the angle between the pericardium and the diaphragm. The subsequent evacuation of the pneumothorax is performed as the telescope is gradually withdrawn, finally together with the trocar. Postoperatively, the catheter is connected to a water-seal drainage bottle and a pressure of $-15 \mathrm{~cm}$ of water is applied for $12 \mathrm{~h}$. The chest tube is removed when subsequent $\mathrm{X}$-ray control has shown the lung to be fully re-expanded.

\section{Discussion}

The timing of therapeutic intervention is primarily dictated by the clinical symptomatology. Special attention has to be directed to the development of the effusion, since a rapid increase can lead to acute tamponade ${ }^{[4]}$. The decision to intervene is supported by the echocardiographic findings. Thoracoscopy not only enables aspiration of recurrent pericardial effusion under visual control, the procedure also facilitates an accurate diagnosis, because it offers possibilities for inspection and guided biopsy of pathological processes ${ }^{[2.5]}$. The accuracy of the latter method should certainly be equivalent to open biopsy, which provides the correct diagnosis in $90 \%$ of the patients suffering from pericardial effusions of malignant origin ${ }^{[6]}$.

The main benefit of endoscopy in a preexisting anatomical cavity is based on the favourable relation between minimal surgical access and anatomical oversight. By employing the video technique, the intervention can be extended to the target organ without additional enlargement of the access and. if indicated, adequate therapeutic fenestration of the pericardial sac can be performed 
simultaneously. The diagnosis (aspiration, biopsy) and therapeutic (fenestration) manoeuvres can be performed under visual control, thus clearly reducing the risk of a heart injury. This technique avoids accidental opening of the abdomen or possible injury to the anterior abdominal wall by intramuscular bleeding; these complications may occasionally occur, however, during surgical procedures using the sub-xiphosternal route ${ }^{[3]}$.

The anatomically more suitable left pleural cavity should be used as the standard access. If the pleural space is obliterated in the area of thoracostomy, as found in our second case, access through the right thoracic cavity offers an alternative. However, fenestration of the pericardial sac from the right side is difficult for several reasons, and not without risk; the pericardium does not protude but lies flat, and the available surface is smaller than on the left side. Furthermore, on the videoscope the right atrium is hard to differentiate in colour from the bluish shimmering pericardium. Injuries to the thin atrial wall may thus occur. The incision of the pericardial sac should therefore be made very carefully in the elevated pericardium. Disorders of cardiac rhythm should be interpreted as a signal that the forceps could have grasped the right atrial wall together with the pericardium. After evacuation of the effusion, step-by-step extension of the incision should be preceded by careful probing of the pericardial cavity, in order to detect potential adhesions between the pericardium and atrium.
The exsudative form of constrictive pericarditis can render the thoracoscopic opening and fenestration impossible, since the obliterative process frequently does not permit the pericardium to be grasped and safely elevated. As illustrated by our third example, in this situation the endoscopic technique may not be considered a safe alternative to open pericardial biopsy, even if there is echocardiographic evidence of pericardial effusion. In addition, the third case emphasizes the importance of the pre-operative echocardiogram, since obliteration of the pericardium is clearly a contraindication to endoscopic techniques.

\section{References}

[1] Callahan JA, Seward JB, Tajik AJ et al. Pericardiocentesis assisted by two dimentional echocardiography. J Thoracic Cardiovasc Surg 1983; 85: 877-9.

[2] Vogel B, Mall W. Thorakoskopische Perikardfensterung diagnostische und therapeutısche Aspekte. Pneumologie 1990; 44: $184-5$.

[3] Little AG, Kremser PC, Wade JL, Levett JM, DeMeester TR, Skinner DB. Operation for diagnosis and treatment of pericardial effusions. Surgery $1984,96 \cdot 738-44$.

[4] Fraser RS, Viloria JB, Wang NS. Cardiac tamponade as a presentation of extracardiac malignancy. Cancer 1980: 45: 1697-1704.

[5] Loddenkemper R. Diagnostık des Pleuraergusses. Internistische Welt 1983, 10: 293-301

[6] Kralstein J, Frishman W. Malignant pericardial diseases: Diagnosis and treatment Am Heart J 1987; 113 785-90. 